\title{
New Explanations of Common Applications and Demonstra- tions of Changes in Pressure with Velocity of a Fluid Current
}

\author{
Xu Changxiang, Zhang Xiaozhong, Chen Youjun \\ Baoyi Group Co. Ltd., Wenzhou, Zhejiang, 325105, China \\ Email address: \\ xchx9763@sina.com (Xu Changxiang), zxz@baoyi.com (Zhang Xiaozhong),info@baoyi.com (Chen Youjun)
}

\section{To cite this article:}

Xu Changxiang, Zhang Xiaozhong, Chen Youjun. New Explanations of Common Applications and Demonstrations of Changes in Pressure with Velocity of a Fluid Current. Special Issue: Xu's Sealing and Flowing Theories of Fluids. International Journal of Energy and Power Engineering. Vol. 5, No. 4-1, 2016, pp. 13-21. doi: 10.11648/j.ijepe.s.2016050401.12

Received: June 22, 2016; Accepted: June 25, 2016; Published: August 24, 2016

\begin{abstract}
Movements are relative. The rapid flowing of a fluid through a wall-bulged passage of pipes at a certain pressure can be regarded as a movement of the bulged wall relative to a static fluid in a certainly pressurized pipe. The axial movement of a cylindrical object in the atmosphere and the water whose free inherent pressure is not influenced can be regarded not only as the object's movement in a certainly pressurized pipe but also as the rapid flowing of a fluid in a certainly pressurized pipe past a static object, because the free inherent pressure (region) is a radial wall and an axial certain pressure of a pipe. Actually, any fluid that flows past an object or a pipe wall at a certain pressure will have a part of its pressure energy converted into its kinetic energy by an axial positive resistance or positive fluid reactance from their windward, and have a part of its kinetic energy converted back into its pressure energy by an axial negative resistance or negative fluid reactance from their leeward, attempting to cause it to flow rapidly past an obstacle met by it without consumption of energy; or any flow of fluids obeys the same mechanism of changes in pressure with velocity and has the same pressure and velocity fields as a flow in a pipe; or it is undoubted that all the common applications and demonstrations of changes in pressure with velocity should have had a uniform scientific explanation.
\end{abstract}

Keywords: Pressure, Velocity, Fluid reactance, Lift, Bernoulli's principle

\section{Introduction}

Any fluid that flows through a sealing joint, on an object and in a pipe is a kind of flowing fluids. They should have had some identical or uniform variables and an equation of variable relationship for describing their flow.

It has been known longest from Bernoulli's principle that the pressure in a fluid decreases as its velocity increases ${ }^{[1]}$. However, it has not been known what are the mechanism and variable equation of the pressure decrease with the velocity increase.

Either Poiseuille's law or Darcy's law only partially relates to variables and variable equations for describing a fluid flow, and cannot alone used to describe a flow of ordinary fluids at all ${ }^{[2]}$.

The air current over a wing flows faster than the air current under the wing, but there has been no uniform convincible interpretation ${ }^{[3 \sim 5]}$. Any ordinary fluid current can rapidly flow past an obstacle almost without any consumption of energy, while a moving solid can only forcibly strike an obstacle by consuming energy; i.e. an ordinary fluid current can avoid its frontal resistance by having a part of its pressure energy converted into its kinetic energy to flow rapidly away ${ }^{[6]}$, whereas any moving solid can only confront its frontal resistance. Because a moving solid will slow down in its original direction and a fluid current will rapidly flow away from its original direction as they meet a frontal resistance, the former movement is in accordance with and the latter flow is not in accordance with the definition that the force $F$ and the acceleration $a$ shall be in the same direction according to Newton's second law $F=m a$. Therefore, the theory of lift from flow turning is invalid, which is proposed on the basis of Newton's second law by NASA Glenn Research Center, and results in another new incorrect explanation of the different airflow speed over and under a wing ${ }^{[7]}$.

Therefore, it is not difficult to understand that there are many incorrect explanations of common applications and demonstrations of changes in pressure with velocity of a fluid current in the prior textbooks and webpages ${ }^{[8]}$. 


\section{New Fundamental Concepts}

\subsection{Steady Flow}

The unit of fluid velocity $[\mathrm{m} / \mathrm{s}]=\left[\left(\mathrm{m}^{3} / \mathrm{m}^{2}\right) / \mathrm{s}\right]$, and so the fluid velocity $v=$ the fluid cubage flowing through a unit cross-section per unit time. Therefore, the fluid current $\left(I_{F}\right)$ defined as the fluid cubage flowing through a cross-section per unit time equals the product of the cross-sectional area $(A)$ and the velocity $(v)$, or the fluid current $I_{F}=A v$ and can be construed as the volume of a fluid cross-section with an area $A$ moving at a velocity $v$ for a unit of time.

If a fluid current without any change in fluid accumulation is called a steady flow, the fluid cubage $\left(I_{F}\right)$ for an incompressible fluid to flow through any cross-section per unit time in a steady flow is constant, or $I_{F}=A v=$ constant. Therefore, in any steady flow of incompressible fluids there is an identical equation of $A_{1} v_{1}=A_{2} v_{2}$ or a fluid velocity $v_{n}$ that changes only with cross section areas $A_{n}$ rather than with time at any cross section, and the greater the cross-sectional area, the slower the velocity, and vice versa; i.e. velocities are not the function of time $(v \neq f(t)$ or $v \neq a t)$ in a steady flow.

Coulomb, using an uncoated disk, a wax-coated disk and a sand-coated disk hung in liquid and having an identical initial turn angle for their free oscillation experiments, verified their attenuations have nothing to do with their surface roughness and only relate to the viscosity of the liquid, or that the frictional resistance of a liquid to an oscillating disk is from the friction between the liquid adhering to the disk and the liquid flowing past the disk ${ }^{[9]}$. In other words, the frictional resistance to a fluid flowing in pipes is from the friction between the static liquid adhering to the pipe wall and the flowing fluid, not directly from the friction between the pipe wall and the liquid. Poiseuille proved that the frictional resistance of a fluid flowing in tubes $R_{F}=(8 \eta l) /\left(\pi r^{4}\right)$ (where $\eta$ $=$ viscosity, $l=$ tube length, $r=$ tube wall radius), which not only has nothing to do with the tube wall roughness but also is not measured in Newton and is the ratio of the pressure $p$ to the fluid current $I_{F}$ between two tube ends, or $R_{F}=p / I_{F}{ }^{[2]}$. However, it has never been used for an ordinary pipe line.

Therefore, it is imaginable that, if the frictional resistance to a fluid flowing in a pipe has nothing to do with its surface roughness, any fluid flowing in a circular pipe will only have such a radial reaction force evenly from its circumferential wall as not to have any radial flowing power, and thus can only flow away in laminar flow without any change in a local accumulation. Of course, any fluid flowing in a circular pipe line will eventually reach a steady flow whether it has one or more steps or thin passages and whether these steps are smoothly transitional or not; i.e. the configuration of steps in a circular pipe line does not influence any steadiness of fluids in the pipe line.

Actually, a fluid flowing in a pipe, when its strength adhering to the pipe wall is not great enough to resist its scour, will be randomly reflected by some wall's micro-rises that are so uncertain in orientation, angle, and magnitude as to cause an axial backflow and a radial flow or a turbulent flow that is asymmetric in both magnitude and deepness, which results in making the fluid resistance relate to the surface roughness of pipe walls.

Since a Reynolds number is the ratio of the inertia force to the viscous force of a fluid, circular pipe walls with different surface roughness and different surface shape symmetry have a different fluid inertia-reflecting ability or a different critical Reynolds number of laminar flow, or a pipe wall to be used under a Reynolds number greater than an ordinary critical Reynolds number can require special surface roughness and surface shape symmetry to avoid a turbulent flow.

\subsection{Mechanism of Changes in Pressure with Velocity}

Theoretically, the kinetic energy of a fluid results from its pressure doing work to its static body, or in a flowing fluid its kinetic energy $0.5 m v^{2}=p A l \rightarrow 0.5 \rho v^{2}=p$, where $m=$ fluid mass, $v=$ fluid velocity, $\rho=m /(A l)=$ fluid density, $p=$ pressure that did work to a static fluid or the pressure that was used to give the fluid the kinetic energy and does not exist in the fluid flowing at the said velocity $v$, and $l=$ distance through which the fluid cross-section with area $A$ is moved by force $p A$. Therefore, any fluid has a conversion of pressure into kinetic energy as it is from its rest to its flow, a conversion of kinetic energy into pressure as it is from its flow to its rest, and an unceasing mutual conversion of pressure and kinetic energy in its velocity-changing flow, always having a pressure decrement or increment equal to a kinetic energy increment or decrement in a unit cubage of fluid.

It is because the pressure's unit $\mathrm{Pa}=\mathrm{N} / \mathrm{m}^{2}=\mathrm{N} \bullet \mathrm{m} / \mathrm{m}^{3}=$ the energy stored in a unit cubage of fluid and the kinetic energy in a unit cubage of fluid is $0.5 \rho v^{2}$ that the mutual conversion of pressure and kinetic energy in a fluid is a formal conversion of two kinds of equivalent energy contained in the fluid, and a speed increase and a pressure decrease arise at the same time as the pressure (energy) is converted into the kinetic energy, and vice versa.

From the equation that the kinetic energy per unit cubage of fluid $0.5 \rho v^{2}=p=$ the pressure used to impart the kinetic energy to the fluid, it can be seen that:

$$
\begin{aligned}
p & =\frac{\rho v^{2}}{2} \quad(\text { where } \rho=\text { fluid density, } v=\text { fluid velocity }) \\
& =\frac{\rho A v^{2}}{2 A} \quad(\text { where } A=\text { fluid cross-sectional area }) \\
& =\frac{\rho I_{F} v}{2 A} \quad\left(\text { where } I_{F}=\text { fluid current }=A v\right) \\
& =I_{F} X_{F} \quad\left(\text { where } X_{F}=\frac{\rho v}{2 A}=\frac{\rho I_{F}}{2 A^{2}}\right) .
\end{aligned}
$$

The quantity $X_{F}, X_{F}=p / I_{F}$, not only has the same measuring unit $\mathrm{Xu}\left(\mathrm{MPa} \bullet \mathrm{h}^{\bullet} \mathrm{m}^{-3}\right)$ as fluid resistance and leak resistance have ${ }^{[6]}$ but also is a factor causing pressure (energy) to be converted into kinetic energy of fluid without any energy loss. Therefore, the quantity $X_{F}$ should be, according to electric reactance, called fluid reactance of a fluid circuit, and the product $\left(I_{F} X_{F}\right)$ of the fluid reactance $\left(X_{F}\right)$ and the fluid current $\left(I_{F}\right)$, called a pressure drop caused by the fluid reactance. 
Therefore, the mechanism of changes in pressure with velocity in a fluid states the pressure in a fluid decreases (increases) as its velocity increases (decreases), where the decrement (increment) of pressure (energy) equals the increment (decrement) of kinetic energy, and a total kinetic energy per unit cubage of fluid $\left(0.5 \rho v^{2}\right)$ equals the product of $I_{F}$ and $X_{F}$, or $0.5 \rho v^{2}=I_{F} X_{F}$, which is the mobility or the pressure drop needed for a fluid current $I_{F}$ to flow past a fluid circuit with a fluid reactance $X_{F}=(\rho v) /(2 A)$, where $\rho=$ fluid density, $v=$ fluid velocity, and $A=$ cross-sectional area of a flow current.

From $\left[0.5 \rho v^{2}=p\right] \rightarrow\left[v=(2 p / \rho)^{0.5}\right] \rightarrow[v \neq f(t)$ or $v \neq a t]$, it can be seen that the kinetic energy $\left(0.5 \rho v^{2}\right)$ in a unit cubage of fluid results from a reactance-caused transient strike of a pressure $p$ on the fluid, or any fluid velocity is from a reactance-caused transient conversion of pressure (energy) into kinetic energy rather than from any pressure gradient in a fluid current at the said velocity because the striking pressure has been devoured by the fluid during the strike. Therefore, any new doctrine ${ }^{[7 \sim 8]}$ is incorrect that is based on Newton's second law to explain that the pressure in a fluid decreases as its velocity increases.

\subsection{Flow of Fluids past a Pipe's Wall-Bulged Passage and an Axially Moving Cylindrical Object}

The pressure $I_{F} X_{F}$ for the fluid mobility is devoured at reducing passages or ports of a pipe line, and the pressure $I_{F} R_{F}$ for the fluid friction is exhausted along a pipe line. Accordingly, the total pressure needed for a fluid to flow in an ordinary pipe line is $I_{F}\left(R_{F}+X_{F}\right)$, or an ordinary pipe line is a series fluid circuit of pipe's fluid resistance and reactance. Therefore, it is not difficult to understand the pressure drop calculated according to Poiseuille's law is only in accordance with the total pressure drop in a capillary line with its fluid speed and reactance negligible rather than in an ordinary pipe line with its fluid speed and reactance not negligible.

Since the kinetic energy possessed by a body theoretically results from a force doing work to its static body and equals the product of the force and the distance through which the force moves the body in the direction of the force, the positive or negative action forces causing a piped fluid to have an increment or decrement of kinetic energy are both in the axial direction of the pipe; i.e. the fluid reactance resulting in a mutual conversion of pressure and kinetic energy in a piped fluid is the axial or frontal positive or negative resistance of reducing passages or ports of a pipe line to fluid. As shown in Figs.1a and 1a', the fluid reactance of the windward (a) piping the fluid into the wall-bulged passage (c) and causing some pressure energy to be converted into kinetic energy in the piped fluid is positive, and the fluid reactance of the leeward (a') piping the fluid out of the wall-bulged passage (c) and causing some kinetic energy to be converted into pressure energy in the piped fluid is negative. Because the reactance $X_{F}$, $X_{F}=\left(\rho I_{F} / 2\right)\left(1 / A_{b}^{2}-1 / A_{a}^{2}\right)$, of a reducing passage (a) is determined by its major and minor cross-sectional areas $A_{a}$ and $A_{b}$, the windward and the leeward that are disposed one after the other and whose major and minor diameters are separately the same always have such a fluid reactance and such a pressure drop or rise equal in magnitude and opposite in direction as to cancel each other out, and even a different shape of the windward or leeward at most causes their equal pressure drop or rise a different pressure gradient. However, the fluid resistance of the windward and leeward cannot cancel each other out, and the total fluid resistance of the wall-bulged passage (c) is the sum of each fluid resistance of its windward (a), leeward (a') and straight (b) passages, where the fluid resistance of the reducer and enlarger can be calculated according to the mean radius of their major and minor ends.

A pipe line is merely made up of some reducing, enlarging and straight passages, and the pressure drop or field of each passage in a certain fluid current can be well known from the said certain fluid current $I_{F}$ and each fluid resistance $R_{F}$ and reactance $X_{F}$ calculated according to their each formulas.

If an axial movement of a cylindrical object in the atmosphere, the river, the lake and the sea does not change their each free inherent pressure, the movement is fully equivalent to a movement of the object in a certainly pressurized pipe and each free inherent pressure (region) is a radial wall and an axial certain pressure of the pipe because the free region only has some axial pressure difference but not any radial pressure difference relative to the moving object and can only cause an axial flow rather than any radial flow of fluids.

Movements are relative. The rapid flow of a fluid through a wall-bulged passage of certainly pressurized pipes can be regarded as a movement of the bulged wall in a static fluid at a certain pressure. The axial movement of a cylindrical object in the atmosphere, the river, the lake and the sea can be regarded not only as the object's movement in a certainly pressurized pipe but also as the rapid flow of a fluid in a certainly pressurized pipe past a static object.

The volume of fluid moved separately by a cylindrical object and a pipe's wall-bulged passage as they each axially move a body length in a static fluid at a certain pressure is equal to the volume of each corresponding right inclusion body whose cross-section is each largest cross-section and whose length is each total length, and thus the axial movement of both a cylindrical object and a pipe's bulged wall in a static fluid at a certain pressure is to cause some static fluid whose cubage equals the volume of their each corresponding right inclusion body to flow between each surface and the static fluid, and not to influence the axial movement of the static fluid in the original position relative to the object and the wall.

Concretely, the flowing of a fluid past a wall-bulged passage of pipes (Figs.1a and 1a') can be regarded as a movement of the bulged wall in a static fluid at a certain pressure, which causes a fluid current increment $\Delta I_{F}, \Delta I_{F}=A$ (the largest cross-sectional area of the bulged wall) $\mathrm{x} v$ (fluid velocity), to hustle between the bulged wall and the static fluid; whereas the axial movement of a cylindrical object (Figs.1b and $1 b^{\prime}$ ) in the atmosphere, river, lake and sea can be regarded as a flow of a fluid in a pipe formed by the free region past a static object, which also causes a fluid current increment $\Delta I_{F}, \Delta I_{F}=A$ (the largest cross-sectional area of the object) $\mathrm{x} v$ (object velocity), to hustle between the object and the static fluid. If the right inclusion bodies corresponding to 
the object and the bulged wall share an axis, a generatrix and a radius equation of $r_{a}=2^{0.5} r_{b}$ (Figs.1c and 1 $\mathrm{c}^{\prime}$ ), their movements relative to fluid are the movement of two right inclusion bodies with the same volume and length relative to a static fluid at a certain pressure, and both to cause a fluid

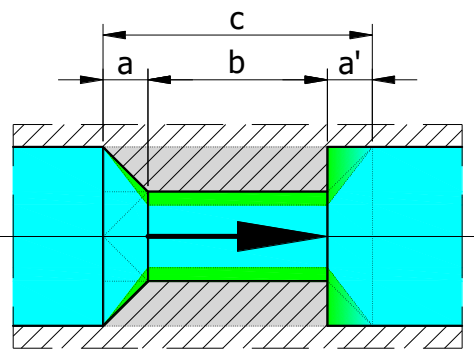

(a) Pipe's wall-bulged passages with a conical windward surface

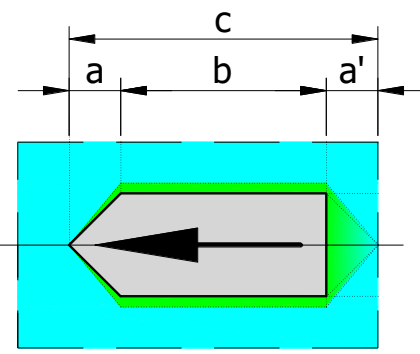

(b) Moving cylindrical objects with a tapered windward surface

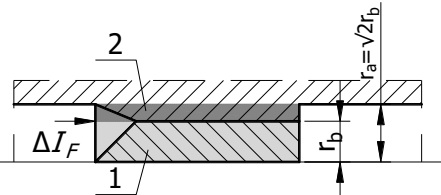

1 Right inclusion body corresponding to an object 2 Right inclusion body corresponding to a bulged wall

(c) The bulged wall and the cylindrical object whose corresponding right inclusion bodies have the same volume current with a similar increment $\Delta I_{F}, \Delta I_{F}=\pi r_{b}{ }^{2} v$, and amount $I_{F}, I_{F}=\pi r_{a}^{2} v=2 \pi r_{b}^{2} v$, to flow past a static object or a pipe's wall-bulged passage with an identical fluid interface, and have the same fluid velocity and pressure fields.

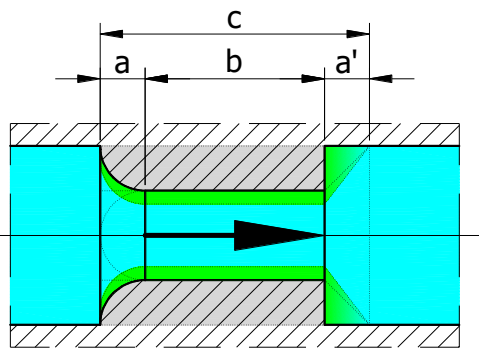

(a') Pipe's wall-bulged passages with an arc windward surface

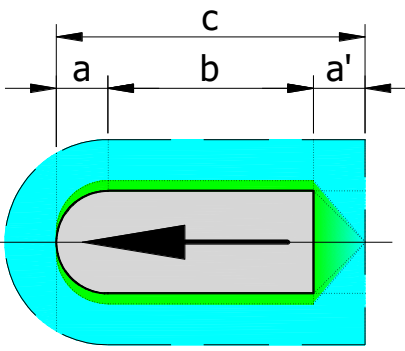

(b') Moving cylindrical objects with a spherical windward surface

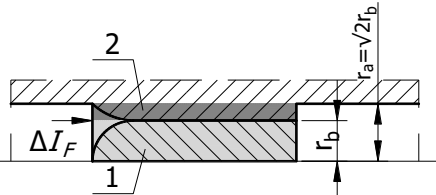

1 Right inclusion body corresponding to an object 2 Right inclusion body corresponding to a bulged wall

(c') The bulged wall and the cylindrical object whose corresponding right inclusion bodies have the same volume

Fig.1 Pipe's wall-bulged passages corresponding to axially moving cylindrical objects

\subsection{Generation and Flow of Air Currents in the Atmosphere}

In their axial and tangent directions, the vanes of a windmill in operation always work as a unit with negative reactance, whereas the vanes of a fan in operation always work as a unit with positive reactance. The windmill vane at first utilizes its axial negative reactance to convert some kinetic energy of coming air currents into some pressure (energy) of slow air flow on its front surface, and then utilizes its tangential negative reactance to convert the pressure (energy) of the slow air flow into the rotational energy of its wheel or spindle. The fan vane at first utilizes its tangential positive reactance to convert some rotational energy of its wheel or spindle into some pressure (energy) of slow air flow on its front surface, and then utilizes its axial positive reactance to convert the pressure (energy) of the slow air flow into the kinetic energy of leaving air currents or blowing winds.

Actually, both a fan wind and a natural wind can be regarded as an air current discharged or ejected out of a pressure vessel. No matter how high pressures they have before being discharged or ejected out, they will become the same pressure as the atmosphere as soon as they are into the atmosphere, which can be verified by a comparison manometer ${ }^{[8]}$. If not at atmospheric pressure, an air current will be continuously radially expanded or shrunk and never flows only in an axial direction. Therefore, the process for air to be discharged or ejected out of pressure vessels is a process for its pressure energy to be fully converted into its kinetic energy, and the higher the pressure before being discharged or ejected out, the higher the speed after being discharged or ejected out, and vice versa.

Any air current in the atmosphere will stop flowing as its kinetic energy is gradually exhausted by the frictional resistance from its periphery. Its flow-stopping process is a process bringing its contiguous atmosphere flowing synchronously with it. If fully vented to the free atmosphere, the contiguous atmosphere will be equivalent to the free atmosphere without any change in velocity and pressure because it can be replenished in time; i.e. the contiguous atmosphere of an air current will be a radial wall and an axial certain pressure of pipes if fully vented to the free atmosphere. If not, the contiguous atmosphere will change in pressure with the velocity of air currents because it cannot be made up in time for 
the brought air. For example, the air on object's leeward that is vented to but not fully to the free atmosphere will flow synchronously with an air current and result in the pressure on the leeward rising along the flow direction from a negative pressure to atmospheric pressure, zero.

\section{Some New Specific Explanations}

\subsection{Flight of Rotational and Irrotational Balls}

The flying rotational ball whose rotational linear velocity

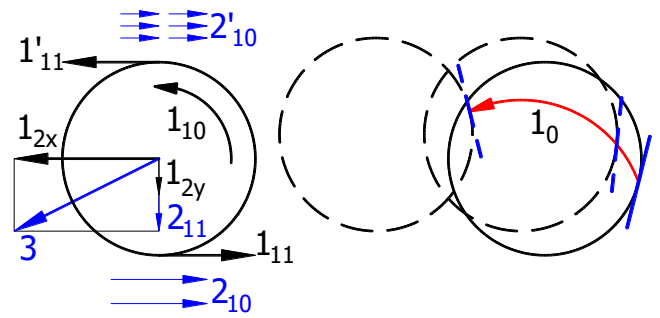

(a) Topspin balls

$1_{0}$ Trajectory of ball-hitting points

$\begin{array}{ll}1_{2 \mathrm{x}} \text { Horizontal flying component } & 1_{2 \mathrm{y}} \text { Vertical flying component free of spin influen } \\ 2_{10} \text { Accelerated flow caused by spin } & 2_{11} \text { Additional pressure difference caused by spin }\end{array}$

$1_{10}$ Rotational direction of balls direction at its upper, lower, left or right pole is the same as its horizontally flying direction is separately called a topspin (Fig.2a), underspin (Fig.2b), left-spin or right-spin ball. The topspin and underspin balls separately have a forward and backward additional roll when they bounce off the ground or the table, and so are also separately called a front-spin or back-spin ball. The side at the pole of which the ball's rotational linear velocity direction is the same as the ball's horizontally flying direction is an upwind side, and the opposite side, a downwind side.

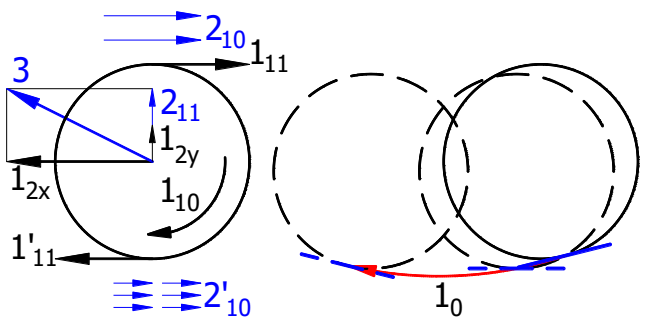

(b) Underspin balls

$1_{11}^{\prime} / 1_{11}$ Rotational linear directions of two poles on a plumb line $2{ }_{10}$ Decelerated flow caused by spin

3 Flying direction with additional pressure difference influence

Fig.2 The hit for and the flight of rotational balls

A ball, if it does not rotate and its friction is ignorable during its flight in the atmosphere, only relates to one resistance from its greatest cross section to airflows past it, which is called its axial resistance to airflows past it. If it rotates during its flight, a ball also relates to a tangential resistance from its outer surface besides having an axial resistance from its greatest cross section. The resistance from the front of the ball's greatest cross section to an airflow past a ball is the axial positive fluid reactance of the ball, and from the back of the ball's greatest cross section, the axial negative fluid reactance of the ball. The resistance from the upwind side of the ball's outer surface to an airflow past a ball is the tangential positive fluid reactance of the ball, and from the downwind side of the ball's outer surface, the tangential negative fluid reactance of the ball. The axial positive fluid reactance of a ball to an airflow past it causes some pressure (energy) to be converted into some kinetic energy of the airflow, and the axial negative fluid reactance causes the kinetic energy to be converted back into the pressure (energy), which results in such a pressure drop on the front of the ball and such a pressure rise on the back of the ball cancelling each other out as not to influence the flight of the ball. The tangential positive fluid reactance of a ball to an airflow past it causes some rotational energy of the ball to be converted into some pressure (energy) of the airflow, and the tangential negative fluid reactance causes some pressure (energy) of the airflow to be converted into some rotational energy of the ball, which is equivalent to transferring some pressure (energy) of the airflow past a rotational ball from its downwind side to its upwind side and making the pressure at its upwind side be greater than the pressure at its downwind side and thus always deflect its flight continuously towards its downwind side. Actually, the upwind side of a rotational ball in flight is a fan wheel (spindle) without vanes, and the downwind side, a windmill wheel (spindle) without vanes. The wheel (spindle), by rotating friction of the flying ball to the airflow past the ball, converts some rotational energy of the ball into some pressure (energy) of the airflow at the upwind side, and converts some pressure (energy) of the airflow at the downwind side into some rotational energy of the ball. Therefore, the rotation of topspin and underspin balls will change in their flying height rather than their flying orientation so continuously as to cause the underspin ball to have a longer hanging time and fly higher and farther than the topspin ball, or cause the topspin ball to have a shorter hanging time and fly lower and nearer than the underspin ball, while they have the same mass, size and initial flight velocity. The rotation of left-spin and right-spin balls will change in their flying orientation rather than their flight height so continuously as to deflect the flight of the left-spin ball continuously towards the right and deflect the flight of the right-spin ball continuously towards the left.

From the above analysis, it can be seen that each additional ascending and descending force of underspin and topspin balls is determined by the velocity difference of resultant airflows from the addition and subtraction of two airflows caused by ball's horizontal flight and rotation separately at the ball's downwind and upwind sides, and any rapidly horizontally rotating ball without flight has no additional ascending or descending force, but a horizontally rotating ball in flight can have a great additional ascending or descending force even when the resultant air current equals zero at its upwind side; i.e. the additional ascending or descending force of horizontally rotational balls in flight is not determined by a 
circulating airflow around them imagined in textbooks.

A topspin ball (Fig.2a) is created by hitting it upward, forward and downward at the middle of its back; an underspin ball (Fig.2b), by hitting it downward, forward and upward at the bottom of its back; a left-spin ball, by hitting it leftward, forward and rightward at the middle of its left; and a right-spin ball, by hitting it rightward, forward and leftward at the middle of its right.

Corresponding to a flying rotational ball, a floating volleyball (Fig.3) is an irrotationally flying ball, which is caused by a transient hitting force through the ball's center (2) of mass and is almost horizontally over the net $\left(5^{\prime} \rightarrow 5\right)$.

Any volleyball inevitably has a non-uniform shell thickness and an air valve that result in its centroid (1) of external outline not coinciding with its center (2) of mass. In the irrotational flight of the volleyball whose center (2) is not the same as its centroid (1), its centroid side has a greater axial fluid reactance to or drag from the airflow past it than its center side, which causes the centroid side to have a greater airflow velocity than the center side, or causes the centroid side to have such a smaller airflow pressure than the center side as to deflect the center's flight towards the centroid side, and at the same time cause the centroid to turn around the center to a steady flight attitude that has the centroid located right behind the center to make the ball have symmetric axial fluid reactance or drag. In the rotational flight of the volleyball whose center is not the same as the centroid, the ball has an equal average axial fluid reactance or drag at the two sides of its flight direction, and does not change in its flight direction.

The axial curving inward and outward vibration of volleyball shells in flight will cause the ball's centroid either to turn or move back and forth relative to the ball's center. The ball's greatest section will change in size so periodically as to cause the ball a periodical change in flight drag or in flight speed when the ball's centroid moves back and forth in the moving direction of the ball's center. The ball will accelerate its deflection towards the right when its vibration deformation can help the fluid reactance or drag to cause its centroid to turn around its center from the right to the dead back of its center, will accelerate its deflection towards the left when its vibration deformation can help the drag to cause its centroid to turn around its center from the left to the dead back of its center, will accelerate its downward deflection when its vibration deformation can help the drag to cause its centroid to turn around its center from the bottom to the dead back of its center, and will accelerate its upward deflection when its vibration deformation can help the drag to cause its centroid to turn around its center from the top to the dead back of its center.

Any volleyball that is hit by a transient force through its center (2) always starts to fly in the direction (5') of the connecting line of its being hit point (4) and its center. In its flight, its asymmetric axial drag always makes its centroid not behind its center turn to the dead back of its center. Therefore, the ball shell's axial curving inward and outward vibration caused by a point contact hit always alternately helps or prevents the ball's centroid not behind the ball's center turn or turning to the dead back of the ball's center. As the vibratory force and the asymmetric axial drag cause the centroid some codirectional turn, the centroid will turn around the center and cause the ball a rapid deflection. As the vibratory force and the asymmetric axial drag cause the centroid some contradirectional turn, the centroid will keep flying not behind the center for a moment. Because each negative amplitude always soon follows a positive amplitude that can help the asymmetric axial drag cause the centroid to turn around the center, the vibratory force and the asymmetric axial drag always co-impart a more float to the flying ball. The ball will mainly float left or right in its flight, if its centroid is on the left or right of the center as the ball is hit. The ball will mainly float upward and downward in its flight, if its centroid is above or below the center as the ball is hit. The ball will mainly periodically accelerate and decelerate in its flight, if its centroid is on the connecting line of its being hit point and its center. Because the position of its centroid relative to its center is random as the ball is hit, each floating ball will random float. If the ball-hitting force is not through the ball's center, the ball will rotate in flight and keep a steady flying attitude that it rotates around a fixed axis without any change in orientation according to the gyroscopic principle, having no floating possibility. If the ball is not served by a transient point contact hit, firstly the hit by a surface contact will cause it such a smaller deformation and secondly the other hit by a long contact will cause it such an increased damping of vibration start, or both cause its centroid such a smaller amplitude, that neither cause it a vibration enough to cause or help its centroid to turn. If the ball does not fly horizontally over the net, it will have no horizontal velocity and drag enough to cause it to float. The volleyball horizontally over the net requires definite ball-hitting height and strength, and thus there is only an overhead float serve. A table tennis ball is so smaller than a volleyball as not to have a misalignment of its centroid and its enter enough to cause it to float. Therefore, the float ball is a special flight phenomena of irrotational balls that is determined jointly by volleyball's size, structure, court size and net height as well as hitting height, manner and strength.
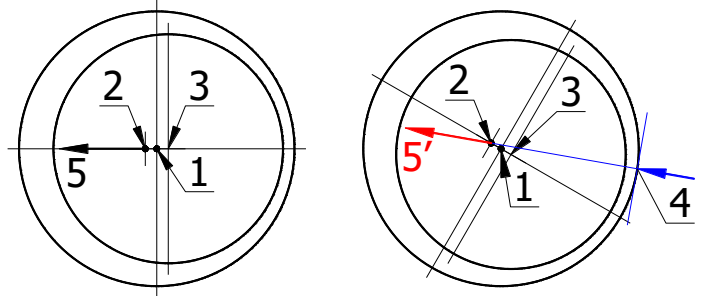

1 Centroid of ext. outline 2 Center of mass 3 Centroid of in. outline 4 Ball-hitting point 5 Steady flight direction 5 ' Unsteady flight direction

Fig.3 The hit for and the flight of floating volleyballs (irrotational)

A golf ball in flight is an authentic underspin ball because it is generated by using an arc swing of club's angular face to slice the bottom of the ball's back side. In comparison, a smoothed golf ball in flight has a small rotating skin surface and friction in air, while a dimpled golf ball in flight has a great rotating skin surface and friction in air. Therefore, the dimpled golf ball can transfer more airflow pressure (energy) 
from its top surface (downwind side) to its bottom surface (upwind side) than the smoothed golf ball and make the former have ascending pressure difference and fly higher and farther than the latter when they have an identical mass, size and slice, which results from a good utilization of ball's rotating friction rather than from saying in textbooks that a more roughed ball has a smaller flight resistance.

\subsection{Lift of Wings}

A wing is an unrolled object of two parallel contiguous pipe lengths placed one under the other. The chord and the upper and lower median curves of a wing's cross-sectional profile separately form the longitudinal section profiles of the two upper and lower pipe lengths. The length of wing chord plane is the circumferences of inlet and outlet cylinders of the two pipe lengths. The two pipe lengths have the same inlet and outlet bores for air and thus the same fluid currents, but have such a different longitudinal section profile and thus have such a different axial fluid resistance and reactance distribution as to have a different axial pressure distribution. The lift of wings results from the inner pressure difference of the two upper and lower pipe lengths.

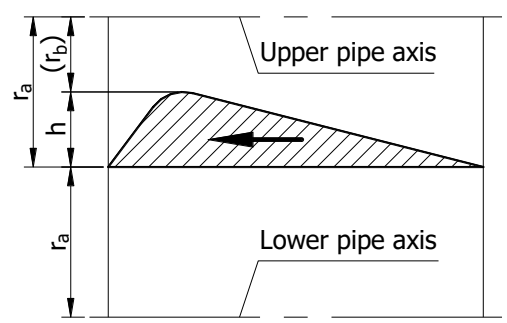

Fig.4 Upper and lower pipe lengths corresponding to a wing

As for a half-cambered wing (Fig.4), whose upper surface is cambered and whose chord plane is its lower surface, it is equivalent to two parallel contiguous pipe lengths whose upper length is one wall-bulged passage of pipes with a windward followed by a leeward and whose lower length is one straight passage of pipes, its mean greatest camber $(h)$ being the greatest wall thickness of the upper pipe length and its length being the circumference of inlet and outlet cylinders of the upper and lower pipe lengths. Because the upper and lower pipe lengths have an identical inlet and outlet bores $\left(2 r_{a}\right)$, they have an identical fluid current $I_{F}=\pi r_{a}^{2} v$, where $v$ is the velocity of the wing relative to the free atmosphere region. However, because the upper pipe length has a smaller average bore $(2 r)$ or a smaller average inner section area $\left(A=\pi r^{2}\right)$ than the lower pipe length, the former has a greater fluid resistance $R_{F}=(8 \eta l) /\left(\pi r^{4}\right)$ and a greater fluid reactance $X_{F}=(\rho v) /(2 A)$ and thus a greater pressure drop of $I_{F} R_{F}$ and $I_{F} X_{F}$ than the latter, i.e. the pressure in the upper pipe length is more negative than in the lower pipe length, which results in a wing having a certain lift.

\subsection{Lift of Plate Type Kites}

An inclined rigid sheet (Fig.5a) in the wind is an unrolled flat surface of two identical conical opening surfaces that are inversely placed one under the other by sharing a generatrix, the width of the rigid sheet being equal to the circumferential sum of the two sectional circles of the upper and lower conical opening at a section. If wind's blowing of the rigid sheet is regarded as sheet's moving in the air, the upper and lower openings each have an air current from the major to the minor end and equal in magnitude and opposite in direction. As for the wind's blowing of the rigid sheet, the fluid reactance of the lower opening to the passing wind is positive and will convert some pressure energy into some kinetic energy in the passing wind and result in a pressure decrement, from atmospheric pressure zero to a negative pressure, along the flow direction, whereas the fluid reactance of the upper opening to the passing wind is negative and will convert some kinetic energy into some pressure energy in the passing wind and result in a pressure increment, from a negative pressure to atmospheric pressure zero, along the flow direction. Accordingly, the inclined rigid sheet in the wind has an ascending resultant force in the front and has a descending resultant force in the back. Obviously, the sheet's front ascending force will be so greater than the sheet's back descending force, if the sheet's front area is greater than the sheet's back area, as to ensure that the rigid sheet has an enough lift in the wind.

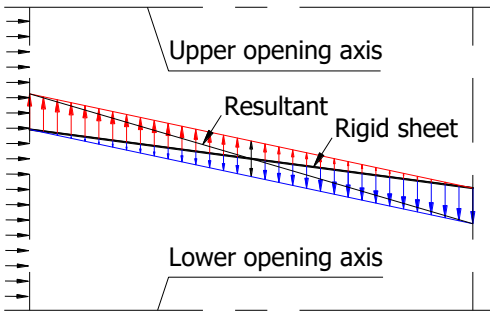

(a) Pressure distribution of inclined rigid sheets in the wind

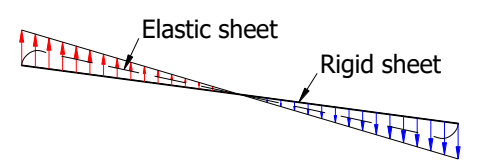

(b) Resultant and deformation of inclined sheets in the wind

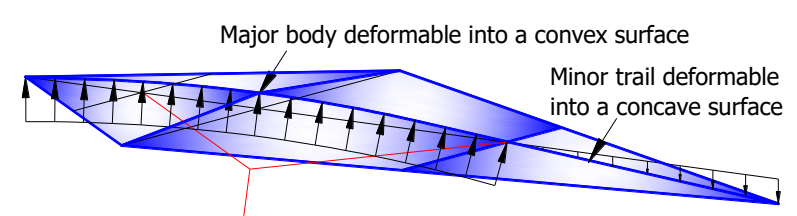

(c) Theoretical mechanical structure of plate type kites

Fig.5 The lift of plate type kites

If an inclined sheet (Fig.5b) in the wind is elastically deformable, the front of the sheet will protrude upward under an ascending resultant force and the back, downward under a descending resultant force. Obviously, the front upward protrusive deformation of the sheet, like a flap added in the front of airfoils, is to decrease the front fluid reactance of the sheet's windward and increase the front ascending force of the sheet, whereas the back downward protrusive deformation of sheet, like a flap added in the back of airfoils, is to increase the back fluid reactance of sheet's windward and the 
back descending force of the sheet, which can help a plate type kite fly upward higher.

Therefore, theoretically plate type kites (Fig.5c) shall have such a rigid framework and an elastic sail as to have a major body that can be deformed into a convex surface and a minor tail that can be deformed into a concave surface in the wind.

\subsection{Atomizing Mechanism of Sprayers}

A sprayer (Fig.6) has a piston cylinder (a) directly connected to an atomizing cavity (b), and a drawing tube that runs from the bottom of liquid container into the atomizing cavity. The cylinder is fully vented directly to the atmosphere via the cavity when the piston is at rest. When the piston moves, the air in the cylinder will be first compressed and then form an airflow flowing through the cavity at a pressure equal to atmospheric pressure $p_{0}$ by converting some pressure energy into some kinetic energy. The airflow will take away some original air in the cavity that is not fully vented to the atmosphere and cause the cavity or the top of the drawing tube to have a pressure lower than the atmospheric pressure $p_{0}$ again by converting some pressure energy into some kinetic energy, thus resulting in the liquid being drawn out of the container into the cavity and blown by the airflow to mist. After ejected out, the mist will gradually stop flowing due to its peripheral friction and stagnate in the atmosphere for a distance (c) away from the nozzle and freely scatter or fall. Therefore, the sprayer's atomization is never caused by the airflow velocity difference inside and outside the atomizing cavity said in textbooks and webpages; in fact, they are two unrelated airflows.

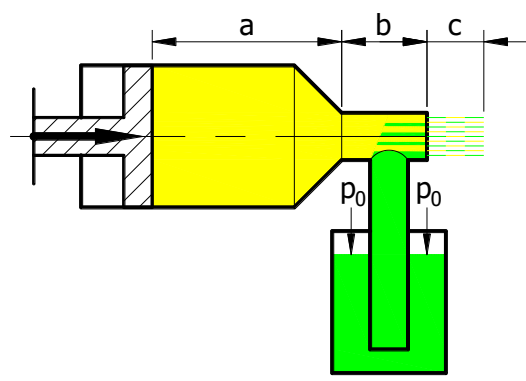

Fig.6 The atomizing mechanism of sprayers

\subsection{False Water Heaters}

What is shown in Fig.7 is a false water heater in textbooks and webpages. The reason for its falsity is that it is never possible for any pipe line that conveys water by a positive pressure to have a thin passage at a negative pressure due to a fast velocity.

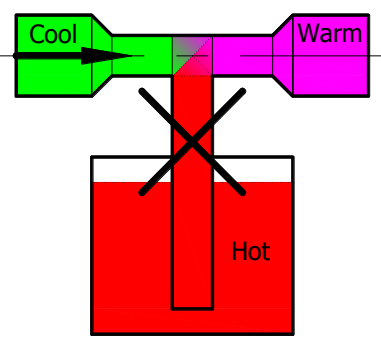

Fig.7 The false water heater
If the thin passage is not at a negative pressure or not at a pressure lower than atmospheric pressure, the water in a water box below the thin passage will be never drawn upward into the thin passage. In the false system shown in Fig.7, the water that fully fills a thin passage will have only a negative power flowing into the atmosphere or have no positive power flowing out of the shower nozzle when the thin passage is at a pressure lower than atmospheric pressure. Actually, it is only when the thin passage in Fig.7 is adequately vented to the atmosphere or directly used as a shower nozzle that a high speed cool water flow ejected from the left can form a negative pressure region between the cool water flow and the wall of the thin passage. However, the construction with a thin passage that is vented to but not fully to the atmosphere has been no longer the construction with a thin passage full of water flow shown in Fig.7.

\subsection{Mechanism for a Blow through Two Pieces of Parallel Hung Paper to Result in Their Mutual Approach}

Any airflow out of a mouth will have its pressure energy in the mouth completely converted into its kinetic energy in the atmosphere or have the same pressure as the atmosphere as soon as it is expelled into the atmosphere. Accordingly, any blow out of a mouth into the space between two pieces of parallel hung paper will never cause any change in the air pressure therebetween when they are so far away from each other that the space between them can be fully vented to the atmosphere. If they are so adequately near to each other (Fig.8) that the original air taken away by the blow cannot be fully replenished in time, the air between them will become so thin as to be at a negative pressure or the blow will convert some pressure energy into some kinetic energy in the air between them and result in a lower pressure there than the atmospheric pressure outside them, thus resulting in them approaching to each other. Therefore, the phenomenon that a blow through two pieces of parallel hung paper results in them approaching to each other is caused by the negative vacuum pressure appearing therebetween when the non-free air between them is taken away by the blow and cannot be fully replenished in time, or absolutely not caused by the airflow velocity difference between and outside the two pieces of paper said in textbooks and webpages. From the fact that any thin wall hose full of fluid absolutely has no negative pressure that can cause the hose to become flat due to a fastest inner velocity, it can be seen that the airflows between and outside the two pieces of paper are unrelated.

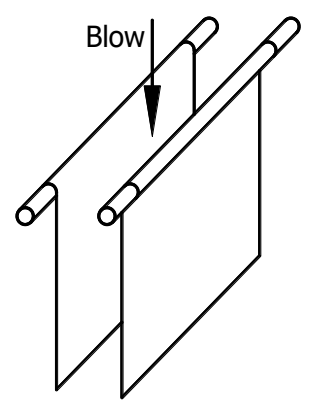

Fig.8 Mechanism for a blow to result in mutual approach of hung paper 
By the way, the mutual approach crash of two parallel running trains or ships near to each other is also caused by train's or ship's taking away the non-free fluid between them, or never by two inner and outer unrelated fluid velocities.

\section{Conclusions}

A flow of fluids through sealing joints and capillaries is so slow as to be able to be regarded as a flow with kinetic energy negligible or only with fluid resistance rather than fluid reactance, and thus pressure drops needed by the flow equal the product of fluid current and leak resistance or fluid resistance.

A general pipe line is to provide a definite flow for fluids, and both its fluid resistance and its fluid reactance need to be considered. Thus pressure drops needed for a fluid to flow through a pipe line equal the product of fluid current and impedance whose value equals the sum of its fluid resistance and reactance.

A flow of fluids past an object is equivalent to a flow of fluids through a pipe's wall-bulged passage corresponding to the object, and thus has the same fluid impedance and pressure drop as the corresponding wall-bulged passage of pipes.

In other words, any flow of fluids through a fluid circuit is determined by its fluid impedance and its pressure difference, where the fluid current through it is directly proportional to the pressure difference between its two ends, and the constant of proportionality is the fluid impedance whose value is equal to the sum of its fluid resistance and reactance. Furthermore, the fluid current flowing through a pipe line is determined by its conveying task; and flowing past an object, by the volume of its corresponding right inclusion body and its moving velocity. The fluid resistance of a pipe line is determined by its geometry and fluid viscosity according to a formula; and the fluid reactance, by its geometry and fluid velocity and density according to another formula. Therefore, according to correspondence of an inner fluid circuit of pipes with an outer fluid circuit of objects, the methods for describing and calculating the change in pressure with velocity in a pipe line can be used to describe and calculate the change in pressure with velocity in all fluid circuits, thus making all the common applications and demonstrations of changes in pressure with velocity of fluid currents have a brand-new uniform scientific explanation.

Note: This paper was initially published in Chinese magazine of Hydraulics Pneumatics \& Seals, 2015, Volume 35 (12): $42-48$.

\section{References}

[1] Hydrodynamica. Encyclopedia Britannica. http://global.britannica.com/EBchecked/topic/658890/Hydrodynamica

[2] Poiseuille's Law. The Great Soviet Encyclopedia, 3rd Edition. http://encyclopedia2.thefreedictionary.com/Poiseuille's +law

[3] NASA Glenn Research Center. Incorrect Lift Theory. http://www.grc.nasa.gov/WWW/K-12/airplane/wrong1.html

[4] Ison, David. Bernoulli or Newton: Who's Right About Lift? [J]. Plane \& Pilot, 2006, July 1

[5] Holger Babinsky. How do wings work? [J]. Physics Education, 2003, Volume 38 (6): 497-503

[6] Xu Changxiang. Pressure Energy, Resistance and Reactance in Leak and Flow of Fluids [J]. Hydraulics Pneumatics \& Seal, 2015, Volume 35 (9): 4-10

[7] NASA Glenn Research Center. Lift from Flow Turning. http://www.grc.nasa.gov/WWW/K-12/airplane/right2.html

[8] Weltner, Klaus et al. Misinterpretations of Bernoulli's Law. http://www-stud.rbi.informatik.uni-frankfurt.de/ plass/MIS/mis6.html

[9] Ding Zurong. Fluid Mechanics: Volume I [M]. Beijing: Higher Education Press, 2004 